\title{
On the structure of eigenfunctions corresponding to embedded eigenvalues of locally perturbed periodic graph operators
}

\author{
Peter Kuchment \\ Department of Mathematics, Texas A\&M University \\ College Station, TX, 77843-3368 \\ kuchment@math.tamu.edu \\ Boris Vainberg \\ Mathematics Department, University of North Carolina \\ Charlotte, NC 28223 \\ brvainbe@email.uncc.edu
}

\begin{abstract}
The article is devoted to the following question. Consider a periodic self-adjoint difference (differential) operator on a graph (quantum graph) $G$ with a co-compact free action of the integer lattice $\mathbb{Z}^{n}$. It is known that a local perturbation of the operator might embed an eigenvalue into the continuous spectrum (a feature uncommon for periodic elliptic operators of second order). In all known constructions of such examples, the corresponding eigenfunction is compactly supported. One wonders whether this must always be the case. The paper answers this question affirmatively. What is more surprising, one can estimate that the eigenmode must be localized not far away from the perturbation (in a neighborhood of the perturbation's support, the width of the neighborhood determined by the unperturbed operator only).

The validity of this result requires the condition of irreducibility of the Fermi (Floquet) surface of the periodic operator, which is expected to be satisfied for instance for periodic Schrödinger operators.
\end{abstract}


2000 Mathematics Subject Classification: 05C90, 34B45, 34L05, 34L40, 35P05, 47A10, 58J50.

Keywords: graph, quantum graph, spectrum, embedded eigenvalue, Fermi surface, Floquet theory

\section{Introduction}

Difference equations on graphs and differential equations on quantum graphs, even when they resemble Laplace or Schrödinger operators in many regards, lack one important property of second order elliptic operators, namely uniqueness of continuation. Uniqueness of continuation states that any solution of an elliptic second order equation $A u=0$ that vanishes on an open set, is identically zero. This is known to be an extremely important property with many implications, in particular in spectral theory. It is also known that elliptic equations of higher orders do not necessarily possess such a property 23], which leads to some weird spectral examples as well (e.g., [15]).

This absence of uniqueness of continuation leads for instance to the following possibilities: a periodic "elliptic second order" operator on a graph (quantum graph) with a co-compact action of an abelian group can have non-empty pure point spectrum (bound states) [14; this is an absolute nono in the continuous case, see [15, 25, 29] and references therein. It is easy to explain this effect for instance as follows: assuming that one has a compact graph with an eigenfunction of the discrete Laplacian that has a zero at some vertex, one can attach this graph by that vertex to any other graph and extend the function as zero still keeping it an eigenfunction. This attachment can also be done in a periodic manner. Such constructions yield these "strange" eigenfunctions generated by compactly supported ones. Indeed, it has been shown that all such bound states on periodic graphs are in fact generated by the compactly supported ones [14, 20]. It is interesting to note that Laplace operator on the Cayley graph of an infinite discrete group can even have solely pure point spectrum [5, 9].

Using the described above attachment procedure, one can also easily construct examples when a localized perturbation of a periodic operator does embed an eigenvalue into absolutely continuous spectrum, which is also expected to be impossible in the continuous situation (albeit this is completely proven in dimensions one only [26, 27] with just a single result in higher dimension available [21, 22]). The aim of this paper is to see what can be 
said about the eigenfunctions corresponding to such embedded eigenvalues. We show not only that such an eigenfunction must be compactly supported, but that its support must be contained in a finite width neighborhood of the support of the perturbation, the width dependent on the unperturbed operator only. Thus, effect of the perturbation seems to be of an extremely short range, when being on the absolutely continuous spectrum of the periodic background.

In the next section we introduce the necessary notions and state and prove the main result for the case of periodic combinatorial graphs (Theorem 5). The following section contains formulation and the proof of the analogous result for the quantum graph case (Theorems 8-10). The paper ends with a brief section containing some final remarks.

\section{Combinatorial graph case}

Consider an infinite combinatorial graph $\Gamma$ with the set of vertices $V$ and a faithful co-compact action of the free abelian group $G=\mathbb{Z}^{n}$ (i.e., the space of $G$-orbits is a finite graph). In fact, in this section we can think of $\Gamma$ just as of a discrete set $V$ of vertices; the graph structure is not truly relevant here, albeit the main operators of interest usually come from graphs (e.g., graph Laplacian [4]). Without loss of generality, the reader may think of the graph as a discrete subset of $\mathbb{R}^{n}$ invariant with respect to all integer shifts. We also consider a $G$-periodic finite difference operator $A$ of a finite order acting on $l_{2}(V)$. Here $l_{2}(V)$ is the space of all square summable functions on $\Gamma$ (i.e., on $V)$. The words "finite difference operator of a finite order" mean that the value of $A u$ at any vertex $v$ involves the values of $u$ at a finitely many other vertices (due to periodicity, the number of these vertices is uniformly bounded). Such periodic operators are clearly bounded in $l_{2}(V)$.

We will fix a (finite) fundamental domain $W$ for the action of $G=\mathbb{Z}^{n}$ on $V$.

Consider for instance the graph below that is a $\mathbb{Z}^{2}$-periodic sub-graph of $\mathbb{R}^{2}$, with the fundamental domain $W$ indicated. An example of a periodic difference operator here could be the version of the Laplace operator that subtracts from the value of a function at a vertex its average value at all vertices adjacent to this one: $\Delta f(v)=f(v)-\frac{1}{d_{v}} \sum_{u \sim v} f(u)$, where $d_{v}$ is the degree of the vertex $v$. This operator is clearly a finite difference operator periodic with respect to the group action. 


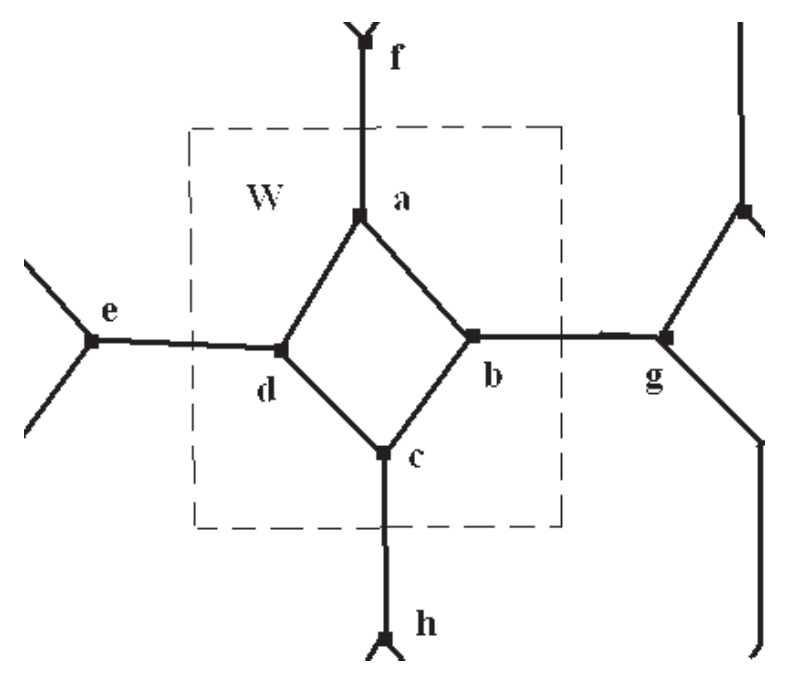

Figure 1: A periodic graph

We will need to measure the sizes of finite subsets $S \subset \Gamma$ by the number and locations of the shifts of the fundamental domain $W$ that are needed to cover $S$. Given a finite subset $S$ of $\Gamma$ we will call its radius the number

$$
r(S)=\min \left\{N \in \mathbb{Z}^{+} \mid S \subset \underset{\gamma \in[-N, N]^{n} \subset \mathbb{Z}^{n}}{\cup} \gamma W\right\} .
$$

We will also need to define two notions of support of a finite difference periodic operator $A$. First of all, let $x$ be a vertex of $\Gamma$. Then we introduce the notion of the $x$-support of $A$ as follows

$$
\operatorname{supp}_{x}(A)=\left\{v \in V \mid\left(A \delta_{v}\right)(x) \neq 0\right\} .
$$

Here $\delta_{v}$ is the delta function on $V$ supported at the vertex $v$, i.e. $\delta_{v}(u)=\delta_{u, v}$ for $u, v \in V$. To put it differently, the $x$-support of $A$ consists of all points $v$, values at which of a function $\psi$ influence the values of $A \psi$ at $x$. We also define the $W$-support of $A$ as

$$
\operatorname{supp}_{W}(A)=\underset{x \in W}{\cup} \operatorname{supp}_{x}(A)=\left\{v \in V\left|A \delta_{v}\right|_{W} \neq 0\right\} .
$$

In other words, the $W$-support of $A$ consists of all points $v$ values at which of a function $\psi$ influence the values of $A \psi$ on $W$. 
As always, dealing with a periodic problem, it is advantageous to use the basic transforms of Floquet theory (e.g., 15, 25]). Namely, for any compactly supported (or sufficiently fast decaying) function $f(v)$ on $V$, we define its Floquet transform as follows:

$$
f(v) \mapsto \hat{f}(v, z)=\sum_{g \in \mathbb{Z}^{n}} f(g v) z^{-g}
$$

where $g v$ denotes the action of $g \in \mathbb{Z}^{n}$ on the point $v \in v, z=\left(z_{1}, \ldots, z_{n}\right) \in$ $\left(\mathbb{C}^{*}\right)^{n}=(\mathbb{C} \backslash 0)^{n}$, and $z^{g}=z_{1}^{g_{1}} \times \ldots \times z_{n}^{g_{n}}$. This is clearly just the Fourier transform on the group $G$ of periods.

One can notice the easily verifiable cyclic (or Floquet) identity

$$
\hat{f}(g v, z)=z^{g} \hat{f}(v, z)
$$

satisfied for any $v \in V$ and $g \in G$. The vector $z$ is sometimes called Floquet multiplier, and if being represented as $z=e^{i k}=\left(e^{i k_{1}}, e^{i k_{2}}, \ldots, e^{i k_{n}}\right)$, vector $k$ is said to be the quasi-momentum (e.g., [2, 15, 25]).

Relation (5) implies that in order to know all the values of the function $\hat{f}(v, z)$, it is sufficient to know them for only one representative $v$ from each $G$-orbit, i.e. for $v$ from a fundamental domain of the $G$-action ${ }^{1}$. Thus, we fix such a fundamental domain $W$ (which is a finite set (graph)) and consider only $v \in W$ in $\hat{f}(v, z)$. We will also denote $\hat{f}(v, z)$ by $\hat{f}(z)$, where the latter expression is a function on $W$ depending on the parameter $z$.

The following statement is immediate:

Lemma 1. The images under the Floquet transform of the compactly supported functions on $\Gamma$ are exactly all finite Laurent series ${ }^{2}$ in $z$ with coefficients in $\mathbb{C}^{|W|}$. Moreover, for a compactly supported function $f$, the Laurent series of $\widehat{f}$ includes only powers $z^{g}$ that satisfy

$$
\|g\|_{\infty}:=\max \left(\left|g_{j}\right|\right) \leq r(\operatorname{supp}(f)),
$$

where $r(S)$ is the radius of a set $S$ introduced in (1).

We will also need the unit torus

$$
\mathbb{T}^{n}=\left\{z \in \mathbb{C}^{n}|| z_{j} \mid=1, j=1, \ldots, n\right\} \subset \mathbb{C}^{n} .
$$

\footnotetext{
${ }^{1}$ In some cases one has to take a more sophisticated approach and treat $\hat{f}(v, z)$ as a section of a naturally defined (depending on $z$ ) line bundle over $\Gamma / G$.

${ }^{2}$ By Laurent series we mean here expansions into powers $z^{g}$ with $g \in G=\mathbb{Z}^{n}$.
} 
It is well known and easy to prove [6, 15, 25] that the Floquet transform (44) extends to an isometry (up to a possible constant factor) between $l_{2}(V)$ and $L_{2}\left(\mathbb{T}^{n}, \mathbb{C}^{|W|}\right)$.

After the Floquet transform, $A$ becomes the operator of multiplication in $L_{2}\left(\mathbb{T}^{n}, \mathbb{C}^{|W|}\right)$ by a rational $|W| \times|W|$ matrix function $A(z)$. To make this clearer, let us compute for the graph shown in Fig. 1 and the Laplace operator $\Delta$ its value $\Delta u$ on a function $u$ that satisfies (5). We notice that for such a function one has $u(f)=z_{2} u(c), u(g)=z_{1} u(d), u(h)=z_{2}^{-1} u(a), u(e)=$ $z_{1}^{-1} u(b)$. Thus, writing the values of $u$ as a vector $(u(a), u(b), u(c), u(d))$, the action of $\Delta$ on $\left.u\right|_{W}$ becomes multiplication by the matrix $A(z)$

$$
\left(\begin{array}{cccc}
1 & -1 / 3 & -1 / 3 z_{2} & -1 / 3 \\
-1 / 3 & 1 & -1 / 3 & -1 / 3 z_{1} \\
-1 / 3 z_{2}^{-1} & -1 / 3 & 1 & -1 / 3 \\
-1 / 3 & -1 / 3 z_{1}^{-1} & -1 / 3 & 1
\end{array}\right)
$$

To formulate our result, we need to introduce another notion.

Definition 2. Let $\lambda \in \mathbb{C}$. We call the Floquet surface $\Phi_{A, \lambda} \subset\left(\mathbb{C}^{*}\right)^{n}$ of the operator $A$ at the energy $\lambda$ the set of all $z \in\left(\mathbb{C}^{*}\right)^{n}$ such that the matrix $A(z)-\lambda$ is not invertible (i.e., $\operatorname{det}(A(z)-\lambda)=0$ ).

The term Floquet surface is non-standard. If one considers quasi-momenta $k$ instead of the Floquet multipliers $z$, one arrives to the standard in solid state physics and theory of periodic equations notion of Fermi surface $F_{A, \lambda}$ 2. 15. So, the Floquet surface is the Fermi one with the natural periodicity with respect to quasimomenta $k$ being factored out.

It is clear from the definition that the Floquet surface is an algebraic subset of dimension $n-1$ in $\mathbb{C}^{n}$. We also look at its intersection with the torus $\mathbb{T}^{n}$

$$
\Phi_{A, \lambda}^{R}=\Phi_{A, \lambda} \cap \mathbb{T}^{n},
$$

which we will call the real Floquet surface. The name comes from the fact that its correspond to real quasimomenta from the Fermi surface.

The following standard fact [6, 15, 25] is easy to prove:

Lemma 3. The point $\lambda$ belongs to the spectrum of the operator $A$ if and only if $\Phi_{A, \lambda}^{R} \neq \emptyset$.

We will also need to introduce some additional notions originating from the solid state physics [2]. Consider for any $z \in \mathbb{T}^{n}$ the operator $A_{z}$ which 
is the restriction of $A$ to the space of all (not square integrable) functions $f$ satisfying the cyclic condition (5). This is a finite dimensional self-adjoint operator that has a finite spectrum $\left\{\lambda_{j}(z)\right\}$, which can be considered as the graph of a multiple-valued function $\sigma\left(A_{z}\right)$. This function is said to be the dispersion relation and its graph the dispersion curve. The preceding Lemma says that the range of this function coincides with the spectrum of $A$ in $l_{2}(\Gamma)$. Arranging the eigenvalues in non-decreasing order splits this curve into continuous (in fact, piecewise-analytic [15, 25, 31]) branches $\lambda_{j}(z)$. Their ranges are finite closed segments of the spectral axis called spectral bands, union of which comprise the whole spectrum $\sigma(A)$. This is the so called band-gap structure of the spectrum [6, 15, 25.

The (complex) Floquet surface is never empty, but when $\lambda$ changes, it moves around. The lemma says that whenever it hits the torus $\mathbb{T}^{n}, \lambda$ belongs to the spectrum. It is natural to expect that when $\lambda$ is a generic point in the interior of the spectrum, then the real Floquet surface will be a variety of the maximal possible real dimension $n-1$ in the torus. This is confirmed by the following statement.

Lemma 4. If $\lambda$ belongs to the interior of a spectral band of the operator $A$, then the real Floquet surface has a part which is a smooth $n$-1-dimensional hyper-surface in $\mathbb{T}^{n}$.

The sketch of the proof of the Lemma (see more details in [21]). Let $\lambda$ belongs to the interior of the band formed by the branch $\lambda_{j}(z)$. Then function $\lambda_{j}(z)-\lambda$ changes sign on $\mathbb{T}^{n}$. Thus, the real Floquet surface separates $\mathbb{T}^{n}$. Now the known analytic structure of the Floquet surface (it is an analytic, or even algebraic in discrete situation set) leads to the conclusion of the lemma (see more details of this part of the argument in [21]).

In what follows, we will need to know that the Floquet surface of $A$ is irreducible as an algebraic variety ${ }^{3}$. This is not always true, but for instance it is conjectured that this is always true if $A$ is the discrete Schrödinger operator on $\mathbb{Z}^{2}$ with potential periodic with respect to a sublattice [8]. This probably is true in any dimension. It was shown in 8 that in $2 D$ irreducibility holds for all but a finitely many of values of the spectral parameter $\lambda$. Examples of some separable cases when irreducibility has been proven can be found in [3, 8, 21, 22.

\footnotetext{
${ }^{3}$ We remind the reader what this means: the variety cannot be represented as the union of two strictly smaller algebraic varieties.
} 
After all this preparation, let us now move to the formulation of the main problem we address in this paper. Consider any local difference operator $B$, i.e. such that its action on a function $u$ involves only the function's values on a finite set $S \subset \Gamma$ and the resulting function $B u$ is supported on $S$ as well. We are interested in the perturbation of the spectrum of $A$ that occurs when the operator is perturbed by adding $B: A+B$. If we assume at this point that $A$ is self-adjoint, it is a general fact that an additional point spectrum might arise (e.g., [25]). It is also the common expectation that this impurity spectrum due to local perturbations should not be embedded into the continuous spectrum of $A$, which is proven for perturbations of a homogeneous background (see the book [7] for a detailed survey). In the case of localized perturbations of a periodic background, absence of embedded eigenvalues is proven for periodic Schrödinger operators in $1 D$ [26, 27. Albeit the same must surely be true in any dimension, the problem in dimensions higher than 1 is hard and only one limited result is known [21, 22]. In the discrete (graph) situation, embedded eigenvalues can arise very easily, due to non-trivial graph topology. Examples of such compactly supported eigenfunctions can be easily constructed using the attachment procedure described before. One might want to ask whether compactness of support of the eigenfunctions corresponding to embedded eigenvalues is the only possibility, and if yes, whether there are any a priori bounds on the size of their supports. Somewhat surprising answer is given by the following result.

Theorem 5. Let $B$ be a local perturbation supported on a finite set $S \subset \Gamma$ (i.e., supp $(B f) \subset S$ for any $f$ ) of a periodic operator $A$. Let $\lambda$ belong to the interior of a spectral band of $A$, the corresponding Floquet surface be irreducible, and $\lambda$ be an embedded eigenvalue for $A+B$. Then the corresponding eigenfunction $f \in l_{2}(V)$ of $A+B$ is compactly supported and moreover,

$$
\left.r(\operatorname{supp} f)<r(S)+r\left(\operatorname{supp}_{W}(A)\right)(2|W|+1)\right) .
$$

The constant $\left.r\left(\operatorname{supp}_{W}(A)\right)(2|W|+1)\right)$ can be usually improved for specific periodic difference operators $A$. (Here $\operatorname{supp}_{W}(A)$ is defined in (2).)

So, the effect of the impurity seems to be of very short range. This theorem will be deduced from the following more general statement:

Theorem 6. Let $\lambda$ belong to the interior of a spectral band of $A$, the corresponding Floquet surface be irreducible, and $\psi$ be a compactly supported 
function on the graph. Assume that the equation $A u-\lambda u=\psi$ has an $l^{2}$ solution $u$. Then $u \in l_{2}(V)$ is compactly supported and moreover,

$$
\left.r(\operatorname{supp} f)<r(\operatorname{supp} \psi)+r\left(\operatorname{supp}_{W}(A)\right)(2|W|+1)\right),
$$

where the constant $\left.r\left(\operatorname{supp}_{W}(A)\right)(2|W|+1)\right)$ can be usually improved for specific periodic difference operators $A$.

Proof of Theorem 6. Since function $\psi$ is compactly supported, its Floquet transform $\widehat{\psi}(v, z)=\sum \psi_{g} z^{g}$ is a Laurent polynomial with degrees $g$ bounded by $\|g\|_{\infty}:=\max _{i}\left|g_{i}\right| \leq r:=r(\operatorname{supp}(\psi))$. Let us denote by e the vector $(1, \ldots, 1) \in \mathbb{Z}^{n}$ and introduce $R:=r\left(\operatorname{supp}_{W}(A)\right)$. We can represent $\widehat{\psi}(z)$ as $z^{-r e} \phi(z)$, where $\phi(z)=z^{r \mathbf{e}} \widehat{\psi}(z)$ is a polynomial that involves only (non-negative) degrees $g \in \mathbb{Z}^{n}$ with $\|g\|_{\infty} \leq 2 r$.

Taking Floquet transform in the equation (10), we rewrite it as

$$
(A(z)-\lambda) \widehat{f}(z)=z^{-r e} \phi(z) .
$$

We can rewrite the Laurent matrix function $A(z)-\lambda$ as $z^{-R \mathbf{e}} A_{1}(z, \lambda)$, where the matrix function $A_{1}(z, \lambda)$ is a polynomial in $z$ involving only the powers $z^{g}$ with $\|g\|_{\infty} \leq 2 R$. Then its inverse can be represented as $z^{R e} \frac{B(z)}{\Delta(z)}$, where $B(z)$ is a matrix polynomial (transposed to the co-factor matrix of $A_{1}$ ) and $\Delta(z)$ is a scalar polynomial (determinant of $A_{1}$ ), which vanishes exactly on the Floquet surface. Thus,

$$
\widehat{f}(z)=z^{(R-r) \mathbf{e}} \frac{B(z) \phi(z)}{\Delta(z)} .
$$

Notice that $B$ involves only powers $z^{g}$ with $\|g\|_{\infty} \leq 2 R(|W|-1)$. We know that $\widehat{f}(z)$ is an $L^{2}$-function on $\mathbb{T}^{n}$. On the other hand, the right hand side of (9) is, up to the factor $z^{(R-r) \mathbf{e}}$, the ratio of two holomorphic polynomials in $\mathbb{C}^{n}$. We also know that zeros of the denominator $\Delta(z)$ in $\left(\mathbb{C}^{*}\right)^{n}$ are irreducible and intersect the torus $\mathbb{T}^{n}$ over an $(n-1)$-dimensional variety. This means that unless the numerator $B \phi$ vanishes on $\mathbb{T}^{n}$ at these zeros to their degrees, the ratio has a singularity that is not square integrable. Thus, the numerator vanishes to that degree, and due to the irreducibility of zeros, the same is true for all zeros in $\left(\mathbb{C}^{*}\right)^{n}$ (see 21] for the details of this simple argument). If there were no zeros of the denominator in $\mathbb{C}^{n} \backslash\left(\mathbb{C}^{*}\right)^{n}$, then, as a corollary of Hilbert's Nullstellensatz, the ratio would be a holomorphic polynomial of 
$z$. We cannot, however, exclude existence of a factor like $z^{q}, q \in\left(\mathbb{Z}^{+}\right)^{n}$ in $\Delta(z)$. If it does exist, we have $\|q\|_{\infty} \leq 2 R|W|$ (since each term in $\Delta$ is like that). Factoring this power out, we represent $\Delta$ as $z^{q} \Delta_{1}(z)$, where zeros of $\Delta$ and $\Delta_{1}$ in $\left(\mathbb{C}^{*}\right)^{n}$ are the same (including their orders), and thus our ratio is a holomorphic polynomial times $z^{-q}$. Notice that division does not increase the degree of a polynomial with respect to any variable. The degree of $\phi(z)$ has been estimated as $\|g\|_{\infty} \leq 2 r$. The additional degree acquired during multiplication by $B$ and division by $\Delta_{1}$ does not exceed $2 R(|W|-1)$. Thus, the ratio $B \phi / \Delta_{1}$ is a polynomial involving the degrees $z^{g}$ with $\|g\|_{\infty} \leq$ $2 r+2 R(|W|-1)$ only. One can calculate now that the effect of the outside factor of $z^{(R-r) \mathbf{e}}$ and of $z^{-q}$ coming from the denominator is to reduce the expression to a Laurent polynomial with degrees $z^{g}, g \in \mathbb{Z}^{n}$ involved with $\|g\|_{\infty} \leq r+R(2|W|+1)$. We see that $\widehat{f}(z)$ is a Laurent polynomial which contains powers of $z$ estimated by $r(\psi)+r\left(\operatorname{supp}_{W}(A)\right)(2|W|+1)$. Reversing the Floquet transform, we get the statement of the theorem.

Let us now address the proof of Theorem [5, which is rather simple. Indeed, the conditions of the theorem imply the equality $A f+B f=\lambda f$, or in a form more convenient for us,

$$
(A-\lambda) f=-B f:=\psi
$$

The function $\psi(v)$ is supported on $S$. Thus Theorem [6 applies and proves the statement.

\section{Quantum graph case}

We now switch to the case of a perturbed periodic quantum graph. We will remind the reader the main definitions concerning quantum graphs. One can find more details concerning quantum graphs in [13, [16]-[20, [24]. A quantum graph $\Gamma$ has each its edge $e$ equipped with a coordinate $x_{e}$ (when no confusion is possible, we use just $x$ instead). This coordinate identifies $e$ with a segment $\left[0, l_{e}\right]$ of the real line. We will also assume that a Schrödinger operator $H=-\frac{d^{2}}{d x^{2}}+V(x), V \in L_{l o c}^{2}(\Gamma)$ with appropriate vertex conditions (all such self-adjoint conditions are described in [11, 13, 18]) is defined on $\Gamma$. The results of this section hold for any of such conditions, however just for simplicity of presentation we will assume that the conditions at each vertex 
are the "standard" Neumann-Kirchhoff ones:

$$
f \text { is continuous and } \sum \frac{d f}{d x_{e}}=0 \text { at each vertex, }
$$

where the sum is taken over all edges incident with the vertex and the derivatives are taken away from the vertex.

As in the previous section, we assume that the graph is acted upon freely and co-compactly by the group $G=\mathbb{Z}^{n}$ that leaves the graph structure (including edges' lengths) and the Hamiltonian $H$ invariant. We use the same letter $W$ as before for a fundamental domain of this action.

One can now introduce the notions of the Floquet transform and Floquet variety of $H$ analogously to the way it was done in the previous section. For instance,

Definition 7. Let $\lambda \in \mathbb{C}$. We call the Floquet surface $\Phi_{H, \lambda} \subset\left(\mathbb{C}^{*}\right)^{n}$ of the operator $H$ at the energy $\lambda$ the set of all $z \in\left(\mathbb{C}^{*}\right)^{n}$ such that the equation $(H-\lambda) u=0$ has a non-trivial solution $u$ that is cyclic with the Floquet multiplier $z$, i.e. such that $u(g x)=z^{g} u(x)$, where $x \in \Gamma$ and $g \in \mathbb{Z}^{n}$. Here, as before, $\mathbb{C}^{*}=\mathbb{C} \backslash\{0\}$.

We can also introduce the same assumption about irreducibility.

The main result of this section is the quantum graph analog of Theorem 5

Theorem 8. Let $w(x) \in L^{2}(\Gamma)$ be supported on a finite set $S$ of edges. Let $\lambda$ belong to the interior of a spectral band of $H$, the corresponding Floquet surface be irreducible, and $\lambda$ be an embedded eigenvalue for $H+w$. Then the corresponding eigenfunction $f \in L_{2}(\Gamma)$ of $H+w$ is compactly supported and moreover,

$$
r(\operatorname{supp} f)<r(\widetilde{S})+C .
$$

Here $C$ is a constant depending on the unperturbed operator $H$ only and for any set of vertices $S$ we define $\widetilde{S}$ as the set of all vertices that are either in $S$, or adjacent to the ones in $S$.

It is possible to give some explicit estimates for the constant $C$, similarly to how it was done for the discrete case. Here, however, the situation depends on whether or not $\lambda$ is the Dirichlet eigenvalue of $H$ on an edge of the graph. 
Theorem 9. Let $w(x) \in L^{2}(\Gamma)$ be supported on a finite set $S$ of edges. Assume that $\lambda$ belongs to the interior of a spectral band of $H$ and is not a Dirichlet eigenvalue on any of the edges, the corresponding Floquet surface is irreducible, and $\lambda$ is an embedded eigenvalue for $H+w$. Then the corresponding eigenfunction $f \in L_{2}(\Gamma)$ of $H+w$ is compactly supported and moreover,

$$
r(\operatorname{supp} f)<r(\widetilde{S})+r(\widetilde{W})(2|\widetilde{W}|+1) .
$$

Here we consider $W$ as a set of vertices.

In case when $\lambda$ does belong to the Dirichlet spectrum of at least one of the edges, the situation is different, and one needs to modify the graph somewhat. We would like to guarantee that $\lambda$ does not contain any points of Dirichlet spectra of $H$ and of $H+w$ on any of the edges of the graph. This is easy to achieve by introducing "fake" vertices. Indeed, if all the edges are sufficiently short, this condition is satisfied. Now, modulo the periodicity, there are only finitely many edges in the graph. Hence, one can introduce a finite set of periodic families of interior points on the edges, such that including these points as new vertices of degree two, one achieves the desired result. Imposing Neumann-Kirchhoff conditions (111) at these vertices (in the case of a vertex of degree two they just mean continuity of the function and its derivative), one makes sure that they do not influence the spectra of $H$ and of $H+w$ at all. This reduces the situation to the case of Theorem 9 , however with an increased number of vertices in the fundamental domain. Let us call this new set $W_{1}$. Then clearly Theorem 9 , if proven, implies the next theorem, and thus also Theorem 8

Theorem 10. Let $w(x) \in L^{2}(\Gamma)$ be supported on a finite set $S$ of edges. Assume that $\lambda$ belongs to the interior of a spectral band of $H$ and is not a Dirichlet eigenvalue on any of the edges, the corresponding Floquet surface is irreducible, and $\lambda$ is an embedded eigenvalue for $H+w$. Then the corresponding eigenfunction $f \in L_{2}(\Gamma)$ of $H+w$ is compactly supported and moreover,

$$
r(\operatorname{supp} f)<r(\widetilde{S})+r\left(\widetilde{W_{1}}\right)\left(2\left|\widetilde{W_{1}}\right|+1\right) .
$$

Proof of Theorem 9 (and therefore also of Theorem 10 and Theorem 8) is based upon its reduction to the discrete version Theorem 5 .

Since we are guaranteed that a neighborhood of $\lambda$ is free of Dirichlet spectra of individual edges, one can use the standard procedure of reducing 
the spectral problems for $H$ and for $H+w$ for the quantum graph to the one for a combinatorial one (e.g., [1, 18, 20]). Namely, assume that one solves the following problem on the graph:

$$
\left\{\begin{array}{l}
-\frac{d^{2} f}{d x^{2}}+V(x) f=\lambda f \text { on each edge } \\
\mathrm{f} \text { is continuous and } \sum \frac{d f}{d x_{e}}=0 \text { at each vertex. }
\end{array}\right.
$$

Since $\lambda$ is not in the Dirichlet spectrum on each edge, one can solve the equation on each edge, assuming the knowledge of vertex values of the function, and then express the Neumann data at the vertices as a linear combination of vertex values. Thus, the only condition that still remains to be satisfied is that the outgoing derivatives at each vertex add up to zero. This clearly will take form of an equation

$$
\sum_{v \sim u} a_{u, v}(\lambda) f(v)=0
$$

satisfied at each vertex $u$ of the graph. One sees that this can be written as a second order difference equation $A(\lambda) f=0$ on the combinatorial graph. Notice that $\operatorname{supp}_{W}(A(\lambda)) \subset \widetilde{W}$. Analogously, the perturbed equation can be rewritten as $A_{1}(\lambda) f=0$. This leads to the two combinatorial counterparts of our periodic and perturbed spectral problems:

$$
A(\lambda) f=0, A_{1}(\lambda) f=0 .
$$

In order to prove the theorem, we will need some simple auxiliary statements collected in the following

Lemma 11. 1. If a function $f$ on the quantum graph satisfies $H f=\lambda f$ (resp. $(H+w) f=\lambda f)$, then its vertex values satisfy the difference equations $A(\lambda) f=0$ (resp. $A_{1}(\lambda) f=0$ ). Conversely, if a vector $f$ of vertex values satisfies $A(\lambda) f=0$ (resp. $A_{1}(\lambda) f=0$ ), it can be uniquely extended to a solution of $H f=\lambda f($ resp. $(H+w) f=\lambda f)$.

2. If the values of such a solution $f$ at both vertices of an edge are equal to zero, then $f$ is zero on this edge. In particular, $f$ is compactly supported if and only if its vertex values are compactly supported, and both supports are of equivalent sizes (i.e., their radii are the same).

3. The operator $A(\lambda)$ is periodic. 
4. The difference operator $B=A_{1}(\lambda)-A(\lambda)$ is supported only on the vertices that are incident to the edges where $w$ has a non-empty support. In particular, $A_{1}(\lambda)$ is a compactly supported perturbation of $A(\lambda)$ with the size of the support of the perturbation controlled by the size of the support of $w$.

5. The Floquet surfaces satisfy the following relation:

$$
\Phi_{H, \lambda}=\Phi_{A(\lambda), 0} .
$$

The proof of the lemma is rather straightforward. Indeed, the way the operators $A$ and $A_{1}$ are defined implies the direct part of the first claim of the lemma. The converse part is also simple. Indeed, if a vector $f$ of vertex values satisfies $A(\lambda) f=0$, let us solve the equation $H u=\lambda u$ on each edge taking $f$ as Dirichlet boundary values (this is possible due to our avoidance of Dirichlet spectra). The resulting function satisfies the equations on the edges and continuity condition by construction. The Neumann condition at the vertices follows now from $A(\lambda) f=0$.

The second claim of the lemma follows from the same avoidance of the Dirichlet spectra.

The third statement follows from periodicity of $H$.

The fourth statement is straightforward from the definitions of $A(\lambda)$ and $A_{1}(\lambda)$.

Let us prove the important (albeit still simple) last statement. If $z \in \Phi_{H, \lambda}$, this means that there exists a non-zero function $f$ satisfying the equation $H f=\lambda f$ and such that $f(g x)=z^{g} f(x)$ for any $g \in \mathbb{Z}^{n}$. This cyclic relation in particularly holds at the vertices, which implies that $z \in \Phi_{A(\lambda), 0}$. Conversely, if $z \in \Phi_{A(\lambda), 0}$, then there is a cyclic vertex function $u$ with the Floquet multiplier $z$ such that $A(\lambda) u=0$. Let us use it as Dirichlet data on each edge to solve $-\frac{d^{2} f}{d x^{2}}+V(x) f=\lambda f$ on each edge. The first claim of the lemma guarantees that we get a solution $f$ of $H f=\lambda f$. We claim that $f$ is cyclic with the Floquet multiplier $z$. Indeed, for any $g \in \mathbb{Z}^{n}$ the functions $f(g x)$ and $z^{g} f(x)$ satisfy the same equation $-\frac{d^{2} f}{d x^{2}}+V(x) f=\lambda f$ on each edge and have the same Dirichlet data. Since we avoided Dirichlet spectrum, we conclude that $f(g x)=z^{g} f(x)$. This proves the Lemma.

We can now finish the proof of the Theorem 8. Indeed, the previous Lemma guarantees that switching from the differential periodic and perturbed problems $H f=\lambda f$ and $(H+w) f=\lambda f$ to the combinatorial problems $A(\lambda) f=0$ and $A_{1}(\lambda) f=0$, one lands in the conditions of Theorem [5] 
Now the same lemma implies that the conclusion of the Theorem 5 about the vertex values implies the conclusion of Theorem 8 about the whole function $f$.

\section{Remarks and acknowledgments}

- The notion of the "radius" $r(S)$ of a finite set in $\Gamma$ depends on the choice of a fundamental domain $W$. Indeed, choosing $W$ further away from $S$ increases $r(S)$. Thus, the optimal way to use the estimates of the main theorems is to choose a fundamental domain $W$ in such a way that $r(S)$ is the smallest possible for a given support $S$ of the perturbation. This would lead to the best localization estimate for the embedded eigenfunctions.

- As it has already been mentioned in the previous section, the NeumannKirchhoff conditions (11) are chosen for simplicity of exposition sake only. Since the results concerning combinatorial graphs are obtained under very general conditions on the periodic operator $A$, the statement of Theorem 8 and its proof carry through if one uses the general selfadjoint vertex conditions (described for instance in [11, 13, 18]). One might have to deal with matrix difference operators $A$, which causes no problem. However, the specific estimates of the constant $C$ of Theorem 8 given in Theorems 9 and 10 will have to change depending on the vertex conditions.

- A deficiency of the results of this paper (as well as of the results of [21, 22 ) is that our technique does not let us treat the case of eigenvalues embedded at spectral edges.

- It is clear from both this paper and [21, 22] that question of irreducibility of the Floquet surface (equivalently, of the Fermi surface, modulo natural periodicity) is intimately related to the problem of existence and behavior of embedded eigenvalues and corresponding eigenfunctions. This does not look like an artifact of the techniques used. It is clear that not arbitrary periodic difference operator satisfies this condition (even higher order periodic elliptic differential operators do not [15). However, it has been proven for discrete Schrödinger operator $-\Delta+V(x)$ on $\mathbb{Z}^{2}$ with potential periodic with respect to a sublattice, 
that irreducibility holds except possibly for finitely many values of $\lambda$ 8]. It was also conjectured in the book [8] that irreducibility always holds for this operator (similar statement is probably correct in any dimension).

Irreducibility is also known for such operators (both discrete and continuous) with separable potentials [3, 8] (see also 21]). It is even sufficient to have in dimension three the potential to separate as $V_{1}\left(x_{1}\right)+$ $V_{2}\left(x_{2}, x_{3}\right)$ [3, 8, 21]. This follows from the results on irreducibility of Bloch variety in dimension two [12].

An advantage of dealing with a difference operator is a possibility of sometimes explicitly computing the determinant $\Delta(z)$ and thus checking its irreducibility.

In fact, examination of the proofs of this text, as well as of [21, 22] shows that we do not need complete irreducibility. What truly required, is that every irreducible component of the Floquet variety intersects the torus $\mathbb{T}^{n}$ over an $n-1$ dimensional set. However, it is not clear how to control this property, and thus it is doubtful that such weaker condition will work better than the full irreducibility.

- As it has been mentioned already, pathologies like pure point spectrum of periodic operators and embedded eigenvalues might and do sometimes appear in discrete or quantum graph situation. However, they do not necessarily have to. Indeed, it is known [14 that the discrete Schrödinger operator $-\Delta+V(x)$ on the lattice $\mathbb{Z}^{n}$ with a potential periodic with respect to a sublattice has absolutely continuous spectrum. This can be proven by the standard L. Thomas' argument 29]. Similarly, there are some cases when one can prove that embedded eigenvalues do not arise from local perturbations of periodic discrete operators. Assume for instance that a difference operator $P$ on the integer lattice $\mathbb{Z}^{n}$ (the operator could in particular be our perturbed operator $A+B$ ) has the following property: there exists an oriented hyperplane $L$ in $\mathbb{R}^{n}$ such that for any point $y \in \Gamma$ there exists a point $x \in \Gamma$ such that $\operatorname{supp}_{x}(P)$ contains the point $y$ and lies completely on the "positive" side of the parallel shift $L_{y}$ to the point $y$, with the only intersection with $L_{y}$ at $y$. Then the equation $P f=0$ has no compactly supported solutions. Indeed, if there were such a solution $f$, consider a support hyperplane $L_{y}$ to $\operatorname{supp}(f)$ such that the whole $\operatorname{supp}(f)$ is on 
the negative side of $L_{y}$ and $y \in \operatorname{supp}(f)$. Consider the point $x$ that serves $y$ as described above. Then the equality $(P f)(x)=0$ clearly implies that $f(y)=0$, which is a contradiction.

This in particular proves the quoted above fact about absence of point spectrum for periodic Schrödinger operators on integer lattices.

- It would be interesting to understand how much the assumption of commutativity of the group of periods influences the validity of the results of this paper. We do not know the answer to this question, but one probably should not expect to be able to go beyond the class of groups of polynomial growth (and hence, according to M. Gromov's result [10], virtually nilpotent ones). Indeed, the results already quoted about the unusual spectral behavior of the lamplighter group (which is of an intermediate growth) [9, 5] show that one might expect surprises there.

- The approach used in this work has been previously used by the authors in different circumstances in [21, 22] (see also [28]). Its idea originates from the paper [30] of the second author.

This research was partly sponsored by the NSF through the Grants DMS0406022 (the first author) and DMS-0405927 (the second author). The authors thank the NSF for this support. The content of this paper does not necessarily reflect the position or the policy of the federal government, and no official endorsement should be inferred.

\section{References}

[1] S. Alexander, Superconductivity of networks. A percolation approach to the effects of disorder, Phys. Rev. B, 27 (1985), 1541-1557.

[2] N.W. Ashcroft and N.D. Mermin, Solid State Physics, New YorkLondon: Holt, Rinehart and Winston 1976.

[3] D. Bättig, H. Knörrer, and E. Trubowitz, A directional compactification of the complex Fermi surface, Compositio Math. 79(1991), no.2, 205229. 
[4] F. Chung, Spectral Graph Theory, Amer. Math. Soc., Providence R.I., 1997.

[5] W. Dicks and T. Schick, The Spectral Measure of Certain Elements of the Complex Group Ring of a Wreath Product, Geometriae Dedicata 93 (2002), 121-137.

[6] M. S. P. Eastham, The Spectral Theory of Periodic Differential Equations, Scottish Acad. Press, Edinburgh - London 1973.

[7] M. S. P. Eastham and H. Kalf, Schrödinger-type Operators with Continuous Spectra, Pitman, Boston 1982.

[8] D. Gieseker, H. Knörrer, and E. Trubowitz, The Geometry of Algebraic Fermi Curves, Acad. Press, Boston, 1992.

[9] R. Grigorchuk and A. Zuk, The lamplighter group as a group generated by a 2 -state automaton, and its spectrum, Geom. Dedicata 87 (2001), 209-244.

[10] M. Gromov, Hyperbolic groups, in Essays in Group Theory, S. M. Gersten ed., MSRI Publ. 8, Springer Verlag, New York, 1987, pp. 75-263.

[11] M. Harmer, Hermitian symplectic geometry and extension theory, J. Phys. A: Math. Gen. 33(2000), 9193-9203

[12] H. Knörrer and J. Trubowitz, A directional compactification of the complex Bloch variety, Comment. Math. Helv. 65(1990), 114-149.

[13] V. Kostrykin and R. Schrader, Kirchhoff's rule for quantum wires, J. Phys. A 32(1999), 595-630.

[14] P. Kuchment, To the Floquet theory of periodic difference equations, in Geometrical and Algebraical Aspects in Several Complex Variables, Cetraro, Italy, 1989, C. Berenstein and D. Struppa, eds.

[15] P. Kuchment, Floquet Theory for Partial Differential Equations, Birkhäuser, Basel 1993.

[16] P. Kuchment, Differential and pseudo-differential operators on graphs as models of mesoscopic systems, in Analysis and Applications, H. Begehr, R. Gilbert, and M. W. Wang (Editors), Kluwer Acad. Publ. 2003, 7-30. 
[17] P. Kuchment, Graph models of wave propagation in thin structures, Waves in Random Media 12(2002), no. 4, R1-R24.

[18] P. Kuchment, Quantum graphs I. Some basic structures, Waves in Random media, 14 (2004), S107-S128.

[19] P. Kuchment, On some spectral problems of mathematical physics, in Partial Differential Equations and Inverse Problems, C. Conca, R. Manasevich, G. Uhlmann, and M. S. Vogelius (Editors), Contemp. Math. v. 362, 2004.

[20] P. Kuchment, Quantum graphs II. Some spectral properties of quantum and combinatorial graphs, J. Phys. A 38 (2005), 4887-4900.

[21] P. Kuchment and B. Vainberg, On embedded eigenvalues of perturbed periodic Schrödinger operators, in Spectral and scattering theory (Newark, DE, 1997), 67-75, Plenum, New York,1998.

[22] P. Kuchment and B. Vainberg, On absence of embedded eigenvalues for Schrödinger operators with perturbed periodic potentials, Commun. Part. Diff. Equat. 25 (2000), no. 9-10, 1809 - 1826.

[23] A. Plis, Non-uniqueness in Cauchy's problem for differential equations of elliptic type, J. Math . Mech. 9 (1960), 557-562.

[24] Quantum Graphs and Their Applications, P. Kuchment (Editor), special issue of Waves in Random Media 14 (2004), no. 1.

[25] M. Reed and B. Simon, Methods of Modern Mathematical Physics, v. 4, Acad. Press, NY 1978.

[26] F. S. Rofe-Beketov, A test for the finiteness of the number of discrete levels introduced into the gaps of a continuous spectrum by perturbations of a periodic potential, Soviet Math. Dokl. $\underline{5}(1964), 689-692$.

[27] F. S. Rofe-Beketov, Spectrum perturbations, the Knezer-type constants and the effective mass of zones-type potentials, in Constructive Theory of Functions'84, Sofia 1984, p.757-766.

[28] W. Shaban and B. Vainberg, Radiation conditions for the difference Schrödinger operators, Applicable Anal. 80 (2002), 525-556. 
[29] L. E. Thomas, Time dependent approach to scattering from impurities in a crystal, Comm. Math. Phys. 33 (1973), 335-343.

[30] B. Vainberg, Principles of radiation, limiting absorption and limiting amplitude in the general theory of partial differential equations, Russian Math. Surveys 21(1966), no 3, 115-193.

[31] C. Wilcox, Theory of Bloch waves, J. Anal. Math. $\underline{33}(1978)$, 146-167. 